\title{
Rectal Carcinoma in a Young Female Patient with Peutz-Jeghers Syndrome: A Case Report
}

\author{
Hsiang-Lin Tsai ${ }^{a, c} \quad$ Chih-Hung Lin ${ }^{b} \quad$ Ya-Lin Cheng ${ }^{a} \quad$ Ching-Wen Huang ${ }^{a}$ \\ Jaw-Yuan Wang a, c, d
}

Departments of a Surgery and ${ }^{b}$ Pathology, and ${ }^{C}$ Cancer Center, Kaohsiung Medical University Hospital, and

d Institute of Clinical Medicine, Kaohsiung Medical University, Kaohsiung, Taiwan, ROC

\section{Key Words}

Peutz-Jeghers syndrome - Rectal carcinoma - Cancer risk .

Hamartomatous polyp $\cdot$ Intussusception

\begin{abstract}
Objective: To report a case of rectal cancer in a patient with Peutz-Jeghers syndrome (PJS). Clinical Presentation and Intervention: A 20-year-old woman with intermittent bloody stool of 4 months was admitted for examination. Gastroendoscopy revealed multiple polyps involving the stomach, small intestine, colon and a rectal adenocarcinoma. A diagnosis of PJS was made based on intestinal polyps with characteristic pathology and melanotic macules on the lips. After surgery and chemotherapy upon follow-up at 8 months, the patient did not have any signs of recurrence. Conclusion: This case showed that rectal carcinoma should be considered for young patients with PJS.

(c) 2013 S. Karger AG, Basel
\end{abstract}

\section{Introduction}

Peutz-Jeghers syndrome (PJS) is an autosomal dominantly inherited syndrome characterized by mucocutaneous pigmentation, gastrointestinal polyps and elevated

\begin{tabular}{ll}
\hline KARGER & $\begin{array}{l}\text { ( } 2013 \text { S. Karger AG, Basel } \\
1011-7571 / 14 / 0231-0089 \$ 39.50 / 0\end{array}$ \\
E-Mail karger@karger.com & $\begin{array}{l}\text { This is an Open Access article licensed under the terms of the } \\
\text { www.karger.com/mpp }\end{array}$ \\
$\begin{array}{l}\text { Creative Commons Attribution-NonCommercial 3.0 Un- } \\
\text { ported license (CC BY-NC) (www.karger.com/OA-license), } \\
\text { applicable to the online version of the article only. Distribu- } \\
\text { tion permitted for non-commercial purposes only. }\end{array}$
\end{tabular}

cancer risk. The cancer risk is not only for gastrointestinal cancers, but also for cancers at other sites, including the breast, ovary, uterus, cervix, lung, and pancreas $[1,2]$. Most of the polyps which occur in the stomach, small intestine and large intestine are hamartomatous in nature. The potential of neoplastic transformation in PJS, as well as the increased risk of cancer at gastrointestinal and nongastrointestinal sites are both widely recognized. In 2006, Hearle et al. [3] also demonstrated that the colorectum was the most common site of malignancy within the gastrointestinal tract. However, malignant degeneration of polyps of the rectum, small intestine, duodenum, and stomach at a young age and then appearing as an advanced rectal carcinoma is a very rare occurrence [4].

Herein, we report a case of a young patient with PJS who had synchronous rectal adenocarcinoma, multiple polyps in the stomach, small bowel, large bowel, and recurrent episodes of jejunojejunal intussusception.

\section{Case Report}

A 20-year-old female patient presented with a 4-month history of intermittent bloody stool, poor appetite, abdominal pain and body weight loss of about $6 \mathrm{~kg}$ within 4 months. Physical examination revealed multiple café-au-lait pigmented macules on the lips and buccal mucosa (fig. 1a). Colonoscopy demonstrated 2 small 
Fig. 1. a A 20-year-old female patient with multiple brown melanotic macules on the lips and mucosa. b Colonoscopy revealed a large polypoid mass $6 \mathrm{~cm}$ from the anal verge, and the biopsy revealed adenocarcinoma (arrow). c Abdominal computed tomography demonstrated a large mass at the rectum (arrow).
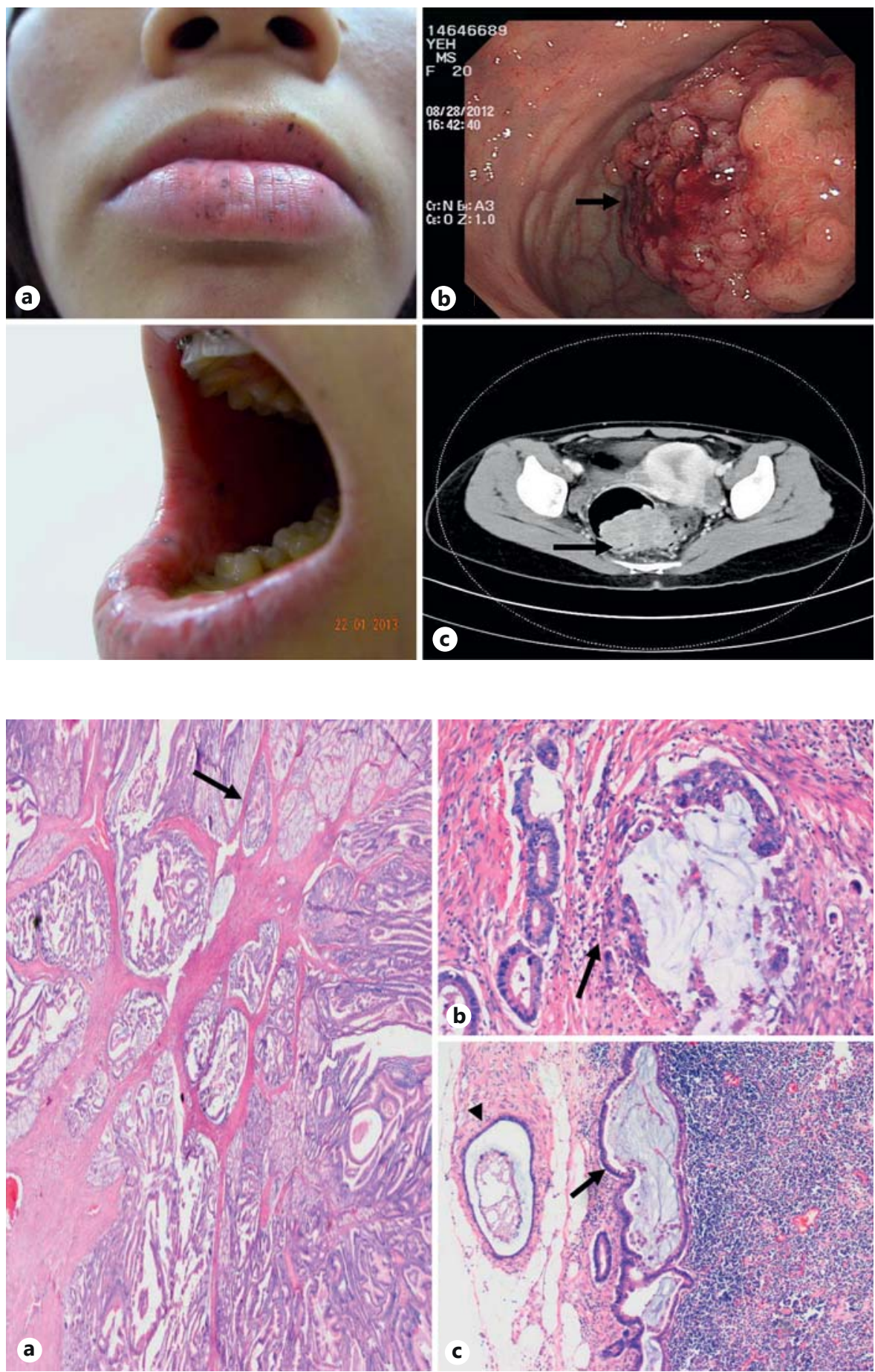

Fig. 2. a Multiple arborizing muscle bundles characteristic of hamartomatous PJS polyps (arrow). $\times 40$. b The lesion also showed invasive nests with pleomorphic cancerous glandular cells microscopically (arrow). $\times 200$. c The retrieved tissue showed lymph node metastases (arrow) and vascular invasion (arrowhead). $\times 40$. polyps 20 and $40 \mathrm{~cm}$ from the anal verge, respectively, but a large mass $6 \mathrm{~cm}$ from the anal verge was also found simultaneously (fig. 1b). Gastroendoscopy showed multiple gastric polyps at the fundus. Biopsies of these colorectal tumors were performed and showed the colonic polyps were villous adenomas and the rectal mass was adenocarcinoma. Abdominal computed tomography re- vealed rectal adenocarcinoma with perirectal invasion and multiple enlarged perirectal lymph nodes (fig. 1c).

Finally, PJS with synchronous rectal cancer was diagnosed. Surgical intervention, i.e. lower anterior resection, of rectal cancer was performed. The size of this lesion was $6 \times 5.7 \mathrm{~cm}$, and histology showed arborizing muscular bundles characteristic of hamar- 
tomatous polyps (fig. 2a), and invasive nests with pleomorphic cancerous glandular cells were detected microscopically (fig. 2b), as were lymph node metastases and vascular invasion (fig. 2c). The grade was 'well differentiated', and 8 of 22 lymph nodes had metastatic adenocarcinoma. The final pathological data revealed T2N2bM0 (UICC stage IIIB). One month after surgery, she suffered from an episode of recurrent jejunojejunal intussusception, and resection of the involved jejunum was performed. Two months after surgery, she was treated with adjuvant chemotherapy (FOLFOX-4) without complications. At the last follow-up 8 months after diagnosis the patient is well and has not signs of recurrent disease assessed with abdominal computed tomography.

\section{Discussion}

In this case, the patient had no history of familial PJS and associated manifestations before the age of 20. Due to this, the diagnosis was delayed and development of malignancy occurred. Malignant degeneration of polyps in the rectum, small intestine, duodenum, and stomach at a very young age and subsequent advanced rectal carcinoma is a very rare occasion [4].

Clinical diagnosis is based on pigmentation and polyp pathology. The pigmentation is particularly in macular form, $1-5 \mathrm{~mm}$ in diameter, and found mostly in the buccal mucosa, on the lips and around the mouth [4]. Polyps associated with PJS are hamartomatous and have smooth muscle arborizing through the polyps, a feature almost unique to PJS.

PJS is caused by a germline mutation in the serine threonine kinase 11 (STK11) gene located on band 19 [4]. About $75 \%$ of patients have an STK11 mutation that can be identified with clinically available testing. Almost all patients with PJS appear to develop signs of the disease (high penetrance). However, there are wide ranges of expression with some patients presenting at a young age with severe symptoms while others only have mild symptoms late in life.
PJS patients require lifelong multidisciplinary care in an attempt to control their significantly elevated cancer risks. The estimated cumulative lifetime risk for developing any cancer in the largest series reports is $87 \%$ by the age of $70[2,3]$. Approximately $15 \%$ of patients had 2 distinct cancers [3]. Unfortunately, due to the elevated cancer risk, the lifespan is shorter than that seen in the general population [5].

Several clinical centers have proposed guidelines for screening individuals with PJS. Most currently advocate upper and lower endoscopy and breast examination, and some advocate surveillance for pancreatic and gynecological malignancies. However, the optimal surveillance strategy for cancer prevention and detection is unclear, and current schemes differ considerably [6].

Most patients have many polyps, ranging in size from several millimeters (sessile) to several centimeters (pedunculated). They are mainly located in the small intestine (78\%), followed by the colon (42\%), stomach (38\%), and rectum (28\%). Polyps are hamartomatous in nature, with bundles of smooth muscle cells. The long-term consequences of the malignancy risk in PJS are significant.

\section{Conclusion}

This case showed that rectal carcinoma should be considered for young patients with PJS.

\section{Acknowledgments}

This work was supported by the foundation Biosignature in Colorectal Cancers, Academia Sinica, Taiwan, and an Excellence for Cancer Research Center Grant funded by the Department of Health, Executive Yuan, Taiwan, Republic of China (DOH102TD-C-111-002).

\section{References}

1 Riegert-Johnson DL, Westra W, Roberts M: High cancer risk and increased mortality in patients with Peutz-Jeghers syndrome. Gut 2012;61:322-323.

2 Giardiello FM, Brensinger JD, Tersmette AC, et al: Very high risk of cancer in familial Peutz-Jeghers syndrome. Gastroenterology 2000;119:1447-1453.
3 Hearle N, Schumacher V, Menko FH, et al: Frequency and spectrum of cancers in the Peutz-Jeghers syndrome. Clin Cancer Res 2006;12:3209-3215.

4 Derici H, Peker Y, Tatar F, et al: Multiple malignant gastrointestinal polyps and rectal carcinoma in a young patient with Peutz-Jeghers syndrome. Int J Colorectal Dis 2007;22:85-86.
${ }_{5}$ You YN, Wolff BG, Boardman LA, et al Peutz-Jeghers syndrome: a study of long-term surgical morbidity and causes of mortality. Familial Cancer 2010;9:609-616.

6 Wirtzfeld DA, Petrelli NJ, Rodriguez-Bigas MA: Hamartomatous polyposis syndromes: molecular genetics, neoplastic risk, and surveillance recommendations. Ann Surg Oncol 2001;8:319-327. 